\title{
Abbautechnologien für zukünftige, mobile Bergbau-Roboter
}

\author{
Michael Berner und Nikolaus August Sifferlinger
}

Lehrstuhl für Bergbaukunde, Bergtechnik und Bergwirtschaft, Montanuniversität Leoben, Leoben, Österreich

Eingegangen 20. Dezember 2021; angenommen 10. Januar 2022; online publiziert 26. Januar 2022

Zusammenfassung: Der folgende Artikel beschäftigt sich mit der Anwendbarkeit unterschiedlicher Abbauwerkzeuge in kleinen, mobilen Bergbau-Robotern. Konventionelle, mechanische Abbauverfahren nehmen einen hohen Stellenwert im aktuellen Untertage-Bergbau ein, jedoch wird deren Effizienz durch die Gesteinsfestigkeit stark eingeschränkt. Die dort entstehenden Reaktionskräfte sind in der Regel hoch und erfordern eine hohe Masse sowie hohe Leistung der Gewinnungsmaschine. Mithilfe zukünftiger Bergbau-Roboter wird versucht, in neue Areale vorzudringen, um Lagerstätten zu erschließen, welche mit herkömmlichen Methoden bis dato als unwirtschaftlich angesehen wurden. Der vergleichsweise kleine Maßstab sowie die geringe Masse dieser Roboter stellen den Einsatz von aktuellen Abbaumethoden vor große Herausforderungen, und es erfordert die Entwicklung neuer Konzepte, um ökonomisch Gestein zu Iösen. Die vielversprechendsten Abbaumethoden werden anhand definierter Parameter verglichen und ihre Anwendung für verschiedene Gesteinsfestigkeiten - unter Berücksichtigung der maximal aufnehmbaren Reaktionskräfte durch die Maschine/den Roboter - bewertet. Auf der Grundlage dieser Studien werden die Durchführbarkeit und Anwendbarkeit bestimmter Abbauwerkzeuge für verschiedene Szenarien und Gesteinsfestigkeiten analysiert. Es ist zu erkennen, dass „klassische“, mechanische Abbaumethoden hinsichtlich der maximalen Druckfestigkeit des Gesteins sehr stark eingeschränkt werden und den dabei entstehenden Reaktionskräfte nicht mehr standgehalten werden kann. Aufgrund dieser Erkenntnisse und unter Berücksichtigung definierter Anforderungen wird eine Konzeptidee eines kombinierten Abbausystems vorgestellt. Die großen Vorteile dieser Technologie sind die vergleichsweise geringen Reaktionskräfte, die verschleißärmere Anwendung und die wasser-hydraulische Energieversorgung. Im ersten Schritt wurden Konzepte

\footnotetext{
M. Berner, Dipl.-Ing. (凷)

Lehrstuhl für Bergbaukunde, Bergtechnik und Bergwirtschaft,

Montanuniversität Leoben,

Franz-Josef-Straße 18

8700 Leoben, Österreich

michael.berner@unileoben.ac.at
}

von mechanischen Bohrgeräten entwickelt und anschließend erste Simulationen des Abbauprozesses mittels DEM durchgeführt, um vorläufige Untersuchungen durchzuführen. Der Beitrag dieser Arbeit soll einen Überblick über die aktuelle Problematik geben und als Basis für künftige Konzeptideen und -entwicklungen dienen.

Schlüsselwörter: Bergbau, Montanmaschinenbau, Abbaumethoden, Robotik, ROBOMINERS

\section{Excavation Methods for Future, Mobile Mining Robots}

Abstract: The following article deals with the applicability of different excavation tools in small, mobile mining robots. Conventional, mechanical excavation methods play a major role in current underground mining, but their efficiency is severely limited by the rock strength. The reaction forces generated there are usually high and require a high weight and high power of the mining machine. With the help of future mining robots, attempts are being made to penetrate new areas in order to develop deposits that were previously considered uneconomical using conventional methods. The comparatively small scale and low weight of these robots pose major challenges to the use of current excavation methods, and the development of new concepts to economically loosen rock is inevitable. The most promising excavation methods are compared on the basis of defined parameters and their application for different rock strengths - taking into account the maximum reaction forces that can be handled by the machine/robot-are evaluated. Based on these studies, the feasibility and applicability of certain excavation tools for different scenarios and rock strengths are analysed. It can be seen that "conventional", mechanical excavation methods are very limited with regard to the maximum compressive strength of the rock and that the resulting reaction forces can no longer be withstood. Based on these findings and considering defined requirements, a concept idea of a combined excavation system is presented. The major advantages of this technology are the comparatively low reaction forces, the low-wear application, and the water-hydraulic energy supply. In the first step, concepts of mechanical drilling tools 
were developed and then first simulations of the excavation process were carried out using DEM to perform preliminary investigations. The article contributes to research by giving an overview of the current problem and serving as a basis for future concept ideas and developments.

Keywords: Mining, Heavy-duty engineering, Excavation methods, Robotics, ROBOMINERS

\section{Einleitung}

Zukünftige Herausforderungen im Bergbau im Hinblick auf Nachhaltigkeit und ökologischen Aspekten erfordern zusätzliche Anstrengungen im Bereich von Forschung und Entwicklung. Mit Hilfe von vollständig automatisierten Maschinen und/oder autonomen Robotern sollen neue Lagerstätten erschlossen oder stillgelegte Bergwerke wieder geöffnet und wirtschaftlich betrieben werden können. Roboter bieten sich für folgende Einsatzgebiete an: Wartung von Maschinen, Erkundung (z. B. von stillgelegten Bergwerken und gefluteten Minen) sowie Vortrieb und Abbau (insbesondere in schwer zugänglichen Bereichen). Die Zukunftsszenarien erfordern neue Ansätze und die Anpassung bestehender Technologien. Veraltete Ansichten müssen unter Umständen ausgeblendet werden, um Raum für neues Denken zu schaffen und innovative Lösungen zu entwickeln, die dazu beitragen, den Bergbau der Zukunft nachhaltiger und wirtschaftlicher zu gestalten. Das Design und die eingesetzten Technologien von Bergbau-Robotern können sich grundlegend von den konventionellen Maschinen unterscheiden, da diese auf die neuen Herausforderungen durch größere Flexibilität und Mobilität angepasst werden müssen. Insbesondere müssen die aktuellen Abbautechnologien mit neuen Standards bewertet werden, um die kommenden Kriterien zu erfüllen [1, 2].

Zahlreiche Forschungs- und Entwicklungsprojekte beschäftigen sich mit der Entwicklung von Robotern für Aufgaben in Umgebungen untertage. Diese Klasse von Robotern ist im Vergleich von Vortriebs- und Abbaumaschinen am anderen Ende der Größenskala. Die vergleichsweise geringe Masse und die geringe verfügbare Leistung sind die limitierenden Faktoren und erfordern daher - neben Anpassungen von kommerziellen Standardprodukten (COTS) - neue Ansätze. Die Interaktion zwischen einem Abbauwerkzeug und dem Gestein erzeugt Kräfte, welche die Maschine aufnehmen bzw. abstützen muss. Das Ausmaß dieser Reaktionskräfte ist je nach Abbaumethode und Gesteinsfestigkeit unterschiedlich.

Abbaumethoden lassen sich in Bohren und Sprengen, mechanische, alternative und kombinierte Abbaumethoden unterteilen, wobei die beiden erstgenannten im modernen Bergbau am häufigsten angewendet werden. Um einen effizienten und wirtschaftlichen Einsatz zu gewährleisten, muss das Abbauwerkzeug eine Reihe von Anforderungen erfüllen, wie z.B. eine angemessene Vortriebsund Abbauleistung, welche maßgeblich durch das abzubauende Gestein beeinflusst werden. Weiters zu beachten sind die Korngrößenverteilung des abgebauten Materials, Verschleißbeständigkeit, generelle Wartungsnotwendig- keiten der Werkzeuge und die Mobilität des Werkzeugs (aber auch der Maschine/des Roboters), die entscheidend für den Abzweigradius der Maschine/des Roboters sind [3, 4].

\section{Limitierungen konventioneller Abbauver- fahren}

Die Leistungsfähigkeit konventioneller, mechanischer Abbaumethoden, wie beispielsweise Teil- oder Vollschnittmethoden, ist einerseits durch die Festigkeit und Abrasivität des abzubauenden Gesteins begrenzt, andererseits auch durch die Größe und Leistung der Maschine limitiert. Bohren und Sprengen stellen klassischerweise eine wirtschaftliche Vortriebs- und Abbaumethode dar, bringen aber ebenso einige nicht zu vernachlässigende Nachteile mit sich. Einige davon sind die Sicherheit während des Lagerungs-, Transport- und Sprengprozesses, Entstehung von giftigen Dämpfen und Gasen sowie Vibrationen und Lärm, Übersprengung, Komplexität der Automatisierbarkeit, diskontinuierlicher Abbau und Schwierigkeiten hinsichtlich automatisiertem/autonomem Sprengen aufgrund der jeweiligen Rechtslage - um nur einige zu erwähnen. Aufgrund dieser Punkte gibt es einen großen Trend im Hinblick auf die Entwicklung vollständig automatisierter, kontinuierlicher Abbaumethoden. Als Schlüsseltechnologien haben sich mechanische Abbaumethoden (Teil- und Vollschnittmethoden) manifestiert. Diese Technologien können sowohl als Vortriebs- und Abbaumethoden eingesetzt werden, sind jedoch (im Speziellen Teilschnittmaschinen) durch die Gesteinsfestigkeit und Abrasivität enorm eingeschränkt. Vollschnittmaschinen mit Schneiddisken können weitaus höhere Gesteinsfestigkeiten bearbeiten, weisen jedoch weitaus geringere Flexibilität und Mobilität als Teilschnittmaschinen auf. Teilschnittmaschinen sind bis zu einem gewissen Grad fähig, enge Kurven und Abzweigungen herzustellen, Vollschnittmaschinen haben im Gegensatz sehr hohe Abzweigradien. Abb. 1 stellt die Flexibilität gegenüber der zu bearbeitbaren Gesteinsfestigkeit mechanischer Vortriebs- und Abbaumaschinen dar und zeigt eine "Lücke" für neue, alternative Technologien auf $[5,6]$.

Die Anwendbarkeit von mechanischen Schneidtechnologien ist zu einem großen Teil von der Gesteinsfestigkeit abhängig. Je höher die Festigkeit eines Gesteins ist, desto höher sind die dafür erforderlichen Schneidkräfte. Diese Informationen sind ausschlaggebend für die grobe Auslegung des Schneidwerkzeuges. Wie in Abb. 1 ersichtlich, sind für Teilschnitttechnologien Festigkeiten bis hin zu $130 \mathrm{MPa}$ wirtschaftlich. Hierbei werden Schneidköpfe, welche mit Rundschaftmeißeln bestückt sind, eingesetzt. Für die Abschätzung der erforderlichen Schneidkräfte gibt es in der Literatur mehrere Ansätze., welche die Schneidtiefe, den Anstellwinkel des Meißels und weitere Parameter berücksichtigen. Nach [7-10] kann die erforderliche Schneidkraft in Abhängigkeit der Druck- und Zugfestigkeit des Gesteins ermittelt werden. In Abb. 2 sind die Schneidkräfte für einen Meißel abhängig von der Druckfestigkeit (Uniaxial Compressive Strength - UCS) dargestellt. 
Abb. 1: Flexibilität gegenüber Leistung mechanischer Vortriebs- und Abbaumaschinen [5]

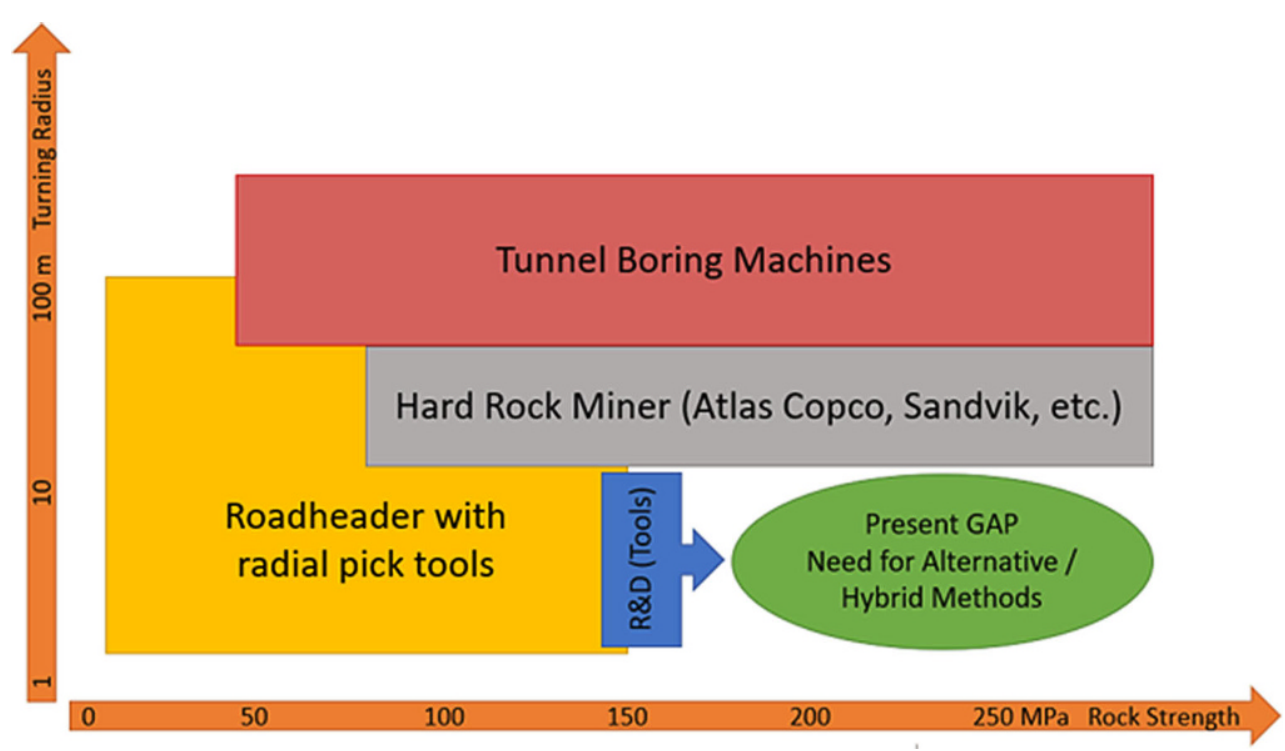

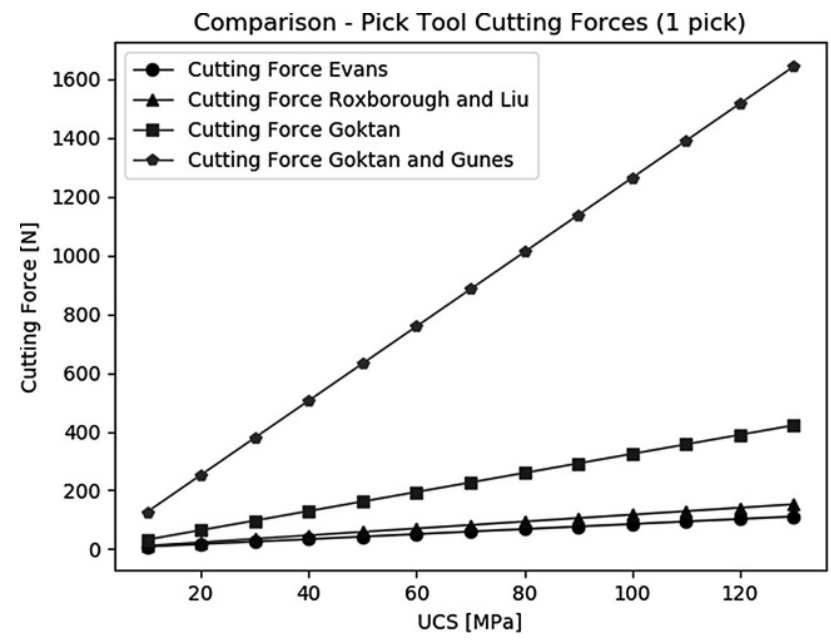

Abb. 2: Erforderliche Schneidkraft eines Rundschaftmeißels [7-10]

Die Reaktionskräfte, welche während des Schneidprozesses entstehen, müssen von der Maschine/dem Roboter aufgenommen werden. Es ist sehr gut zu erkennen, dass bereits für einen Meißel bei moderater Gesteinsfestigkeit die Schneidkräfte im Bereich einiger hundert Newton sind. Beachtet man, dass sich während des Schneidens mehrere Meißel im Eingriff befinden, entstehen insgesamt äußerst hohe Reaktionskräfte.

Tunnelbohrmaschinen und sogenannte Hard Rock Miner nutzten Disken als Schneidwerkzeuge. Mithilfe dieser Schneiddisken können in der Regel höhere Gesteinsfestigkeiten (bis hin zu $300 \mathrm{MPa}$ ) bearbeitet werden. Rostami [11] entwickelte einen Ansatz, die erforderliche Schneidkraft von Disken zu berechnen, indem eine Relation zwischen der Spannungsverteilung in der Kontaktzone und der entstehenden Kraft an der Diske gefunden werden konnte. In Abb. 3 werden die Verläufe der Gesamtkraft sowie deren Anteile in Abhängigkeit der Druckfestigkeit (UCS) für eine Schneiddiske dargestellt.
Der Tatsache, dass Tunnelbohrmaschinen und Hard Rock Miner in Hartgesteinszonen vordringen können, stehen im Vergleich zu Teilschnittmaschinen sehr hohe Kräfte - auch schon bei weichem Gestein - gegenüber.

Diese Ergebnisse zeigen eindeutig auf, dass es bei Einsatz dieser Technologien Maschinen bedarf, welche mit entsprechender Leistung und Masse und etwaigen Verankerungen oder Abstützungen den Reaktionskräften entgegenwirken können müssen.

\section{Herausforderungen}

Wie in Abschn. 2 beschrieben, ist die Wirtschaftlichkeit der mechanischen Abbaumethoden durch die Festigkeit und Abrasivität des zu lösenden Gesteins begrenzt. Wie bereits erwähnt, erfordert der Löseprozess bei mechanischen Abbaumethoden hohe Kräfte, welche wiederum auf die Maschine als Reaktionskräfte einwirken. Kleine Bergbaumaschinen (Bergbauroboter) haben eine begrenzte Leistung und eine vergleichsweise geringe Masse und können somit nur geringe Reaktionskräfte aufnehmen.

Während des Abbauprozesses entstehen durch die Interaktion zwischen Werkzeug und Gestein, welche geringer als die Traktionskräfte der Maschine sein müssen. Dieser Widerstand errechnet sich aus dem Produkt der Schwerkraft der Maschine und des Widerstands- oder Kraftschlussbeiwertes. Je nach Fahrwerk (Typ und Material) und Untergrundmaterial ergeben sich unterschiedliche Widerstandsbeiwerte [12].

Untertage werden typischerweise Gummiluftreifen oder Raupenketten als Fahrwerke von Vortriebs- und Abbaumaschinen eingesetzt. Im Rahmen des EU-Projektes H2020ROBOMINERS wurden weitere Konzepte zur Fortbewegung von Robotern in harschen Umgebungen betrachtet. Erkenntnisse aus dieser Arbeit zeigten das große Potenzial von Schneckenrädern als Antriebsfahrwerke. In Abb. 4 sind die Traktionskräfte der drei oben genannten Varianten für eine Maschine/einen Roboter mit einer Gesamtmasse von 
Abb. 3: Erforderliche Kraft einer Schneiddiske [11]

Abb. 4: Traktionskräfte verschiedener Antriebskonzepte in Abhängigkeit des Untergrundmaterials $[13,14]$
Disc Cutting Forces (1 disc)

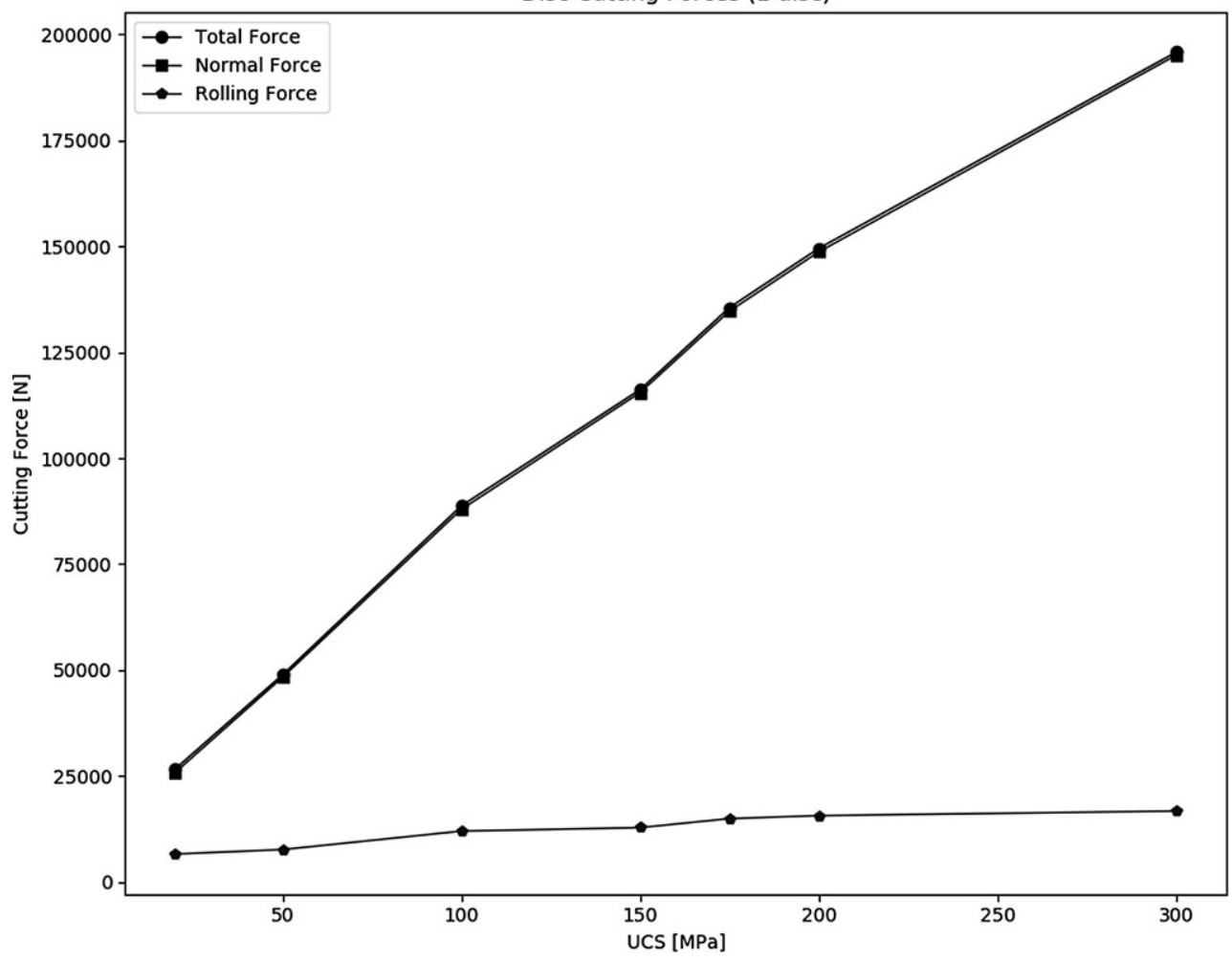

Comparison of traction forces

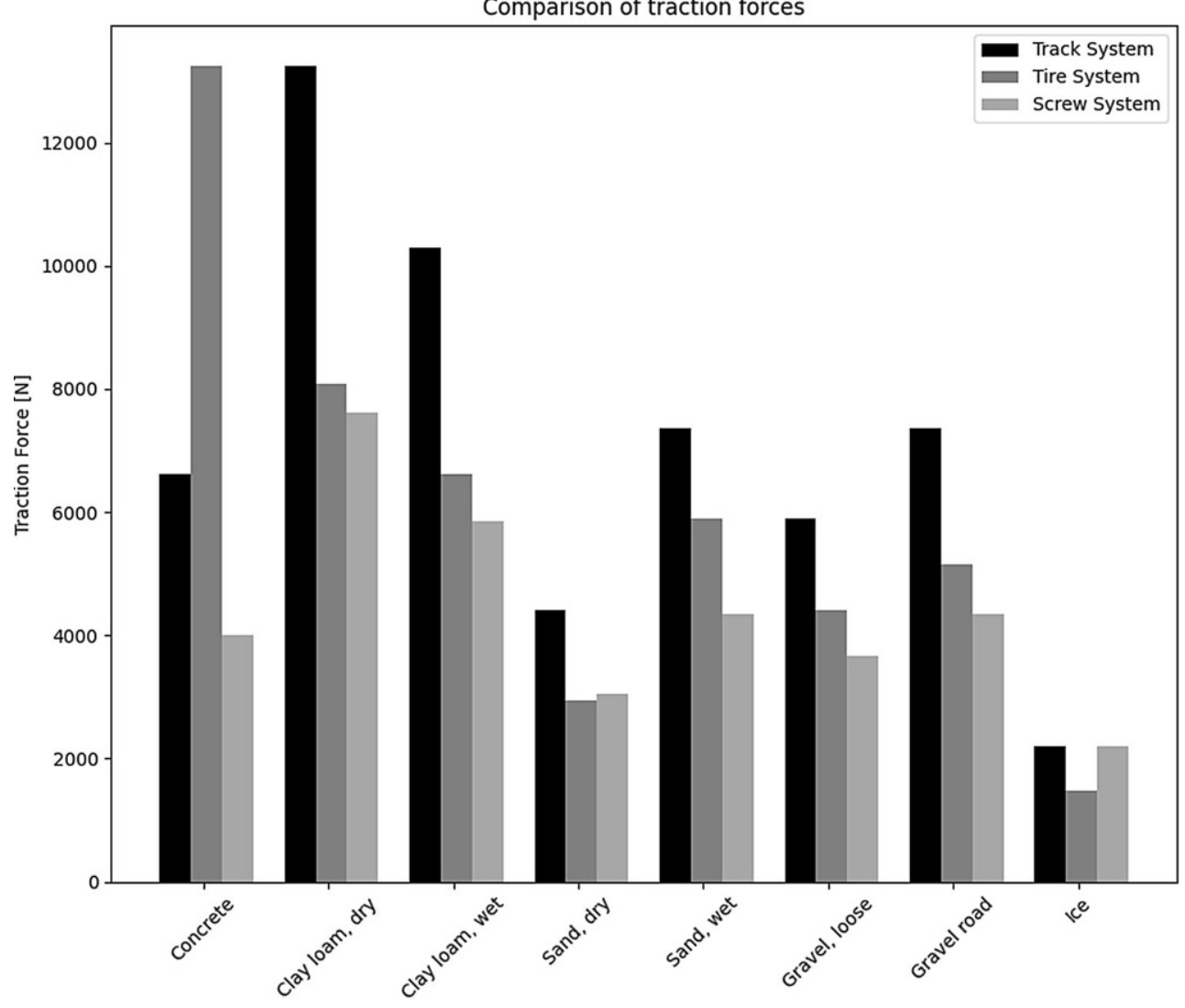


Abb. 5: Traktionskräfte von Schneckenradsystemen mit und ohne zusätzlicher Spannkraft

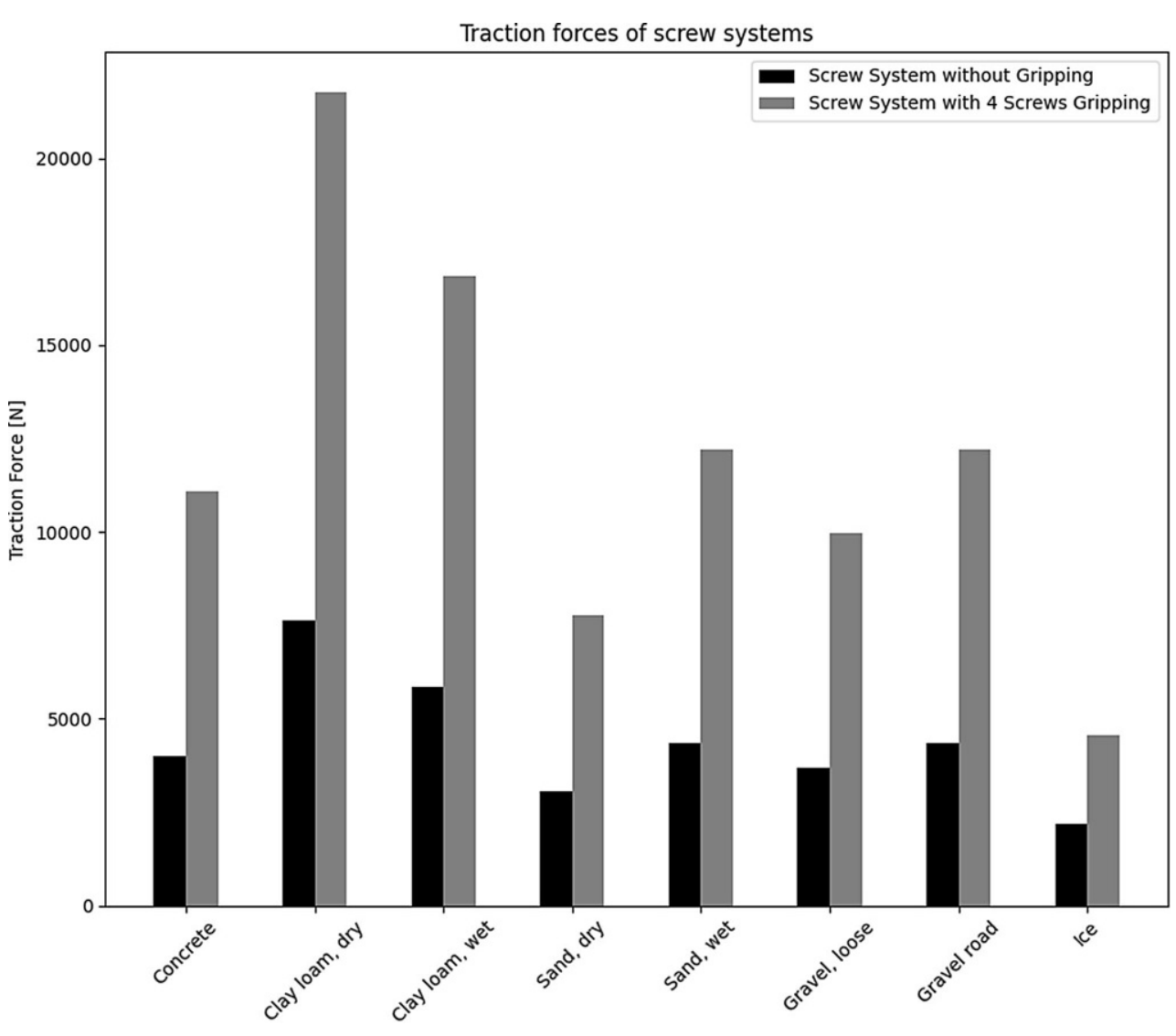

\begin{tabular}{|c|c|c|c|}
\hline Drill and Blast & Mechanical Excavation & Alternative Excavation & Combined Excavation \\
\hline & $\begin{array}{l}\text { - Drilling } \\
\text { - Part-face cutting } \\
\text { - Full-face } \\
\text { - Impact hammer } \\
\text { - Saw cutting } \\
\text { - Grinding } \\
\text { - Auger drilling } \\
\text { - Dredging } \\
\text { - Bucket wheel excavation }\end{array}$ & $\begin{array}{l}\text { - } \text { High-pressure water } \\
\text { cutting } \\
\text { - } \text { Hydrofracturing } \\
\text { - Laser cutting } \\
\text { - Chemical excavation } \\
\text { - Plasma blasting } \\
\text { - }\end{array}$ & $\begin{array}{l}\text { - High-pressure water } \\
\text { assisted to drilling } \\
\text { - High-pressure water } \\
\text { assisted to cutting } \\
\text { - } \text { Microwaves assisted to } \\
\text { cutting } \\
\text { - Ultrasonic drilling }\end{array}$ \\
\hline
\end{tabular}

$1500 \mathrm{~kg}$ gegenübergestellt. Diese Traktionskräfte wurden nach $[13,14]$ ermittelt und graphisch für unterschiedliche Untergrundmaterialien aufbereitet.

Im Allgemeinen weisen Raupenfahrwerke die größten Traktionskräfte auf, Gummiluftreifen besitzen ausschließlich auf Asphalt größere Bodenhaftung und Schneckenräder zeigen in keinem Anwendungsfall einen direkten Vorteil gegenüber den beiden anderen Antriebsarten.

Jedoch können Schneckenräder vielseitiger eingesetzt werden und können somit die Flexibilität und Mobilität einer Maschine/eines Roboters erhöhen. Eine symmetrische Anordnung einer geradzahligen Anzahl an Schneckenrädern in Bezug auf den Grundkörper der Maschine/des Roboters erlaubt erstens einen richtungs- und lageunabhängigen Einsatz und zweitens können durch Beaufschlagung einer zusätzlichen Spannkraft mithilfe der Schneckenräder die Traktionskräfte signifikant erhöht werden (Abb. 5).

\section{Auswahl alternativer Abbauverfahren}

Um die Anwendbarkeit der in Frage kommenden Abbaumethoden zu beurteilen, müssen sie vorab klassifiziert und grob analysiert werden. Generell können Abbaumethoden unterteilt werden in: Bohren und Sprengen, mechanische, alternative und kombinierte Abbaumethoden (Abb. 6) [5, 6, $15,16]$.

In [6] wurden diese Technologien nach ausgewählten Kriterien untersucht, analysiert und bewertet. Viele dieser Methoden werden für die Gewinnung von Rohstoffen oder für den Tunnelbau eingesetzt, aber nicht alle sind in einem kleinen Maßstab wirtschaftlich. Zunächst werden die folgenden Merkmale bewertet, die für künftige Abbauwerkzeuge kleiner, mobiler Bergbauroboter zwingend erforderlich sind: 


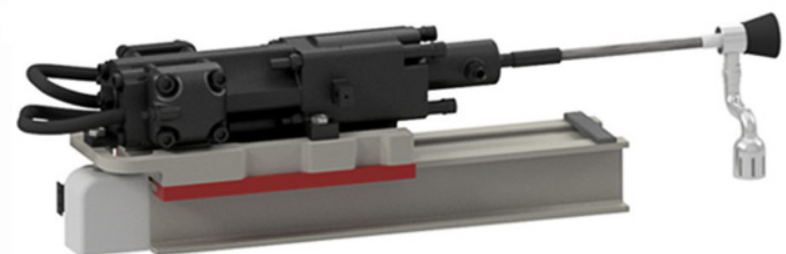

a

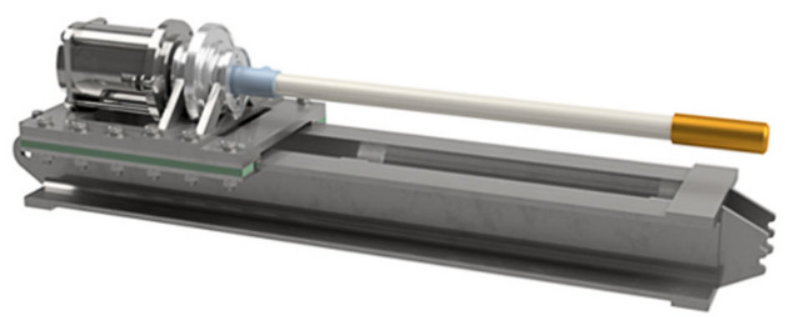

C

Abb. 7: Konzepte Bohrgeräte
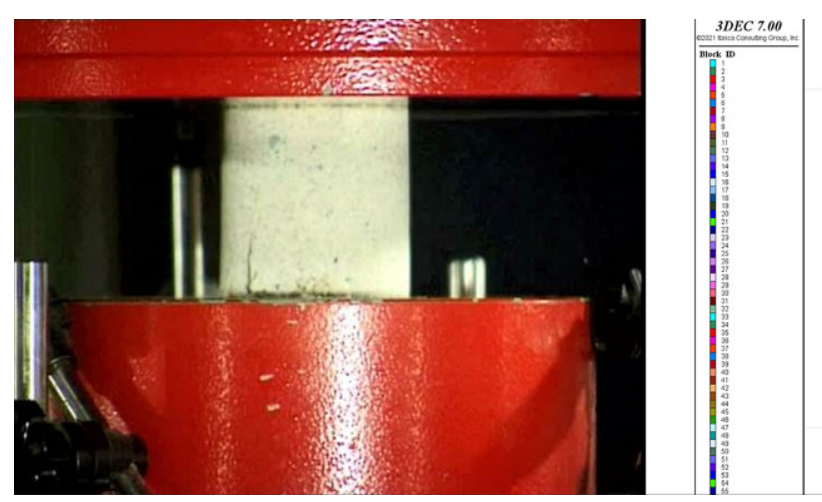

Abb. 8: Kalibrierung der Materialparameter

- Die Fähigkeit, kontinuierlich Material abzubauen.

- Die Fähigkeit, Tunnel für die eigene Fortbewegung der Maschine/des Roboters zu erstellen.

- Einschränkungen (Druckfestigkeit und Abrasivität des abzubauenden Gesteins usw.)

Bohren und Sprengen ist aufgrund seiner allgemeinen Anwendbarkeit im Berg- und Tunnelbau und seiner hohen Produktionsrate (vor allem bei Hartgestein) eine der am häufigsten eingesetzten Vortriebs- und Abbautechnologien. Mechanische Abbausysteme sind im Vergleich zu Bohren und Sprengen mindestens ebenso populär und weisen einige Vorteile auf, wie z. B. einen sichereren Betrieb, eine bessere Fähigkeit zum selektiven Abbau und den kontinuierlichen Materialabbau. Alternative Abbausysteme umfassen neben dem mechanischen Abbau und Bohren und Sprengen auch nicht-konventionelle (alternative) Abbaumethoden. Haupteinsatzgebiete sind Präzisionsaufgaben, Vorschwächung des abzubauenden Gesteins (bei kombinierten Abbausystemen) und Aufgaben, bei denen die Umgebungsbedingungen konventionelle Verfahren nicht zulassen. Kombinierte Abbausysteme vereinen die Vorteile von mechanischen Abbausystemen und alternativen Abbaumethoden. Hilfswerkzeuge liefern einen zusätzlichen Energieeintrag, um das Gestein vorzuschwächen oder die

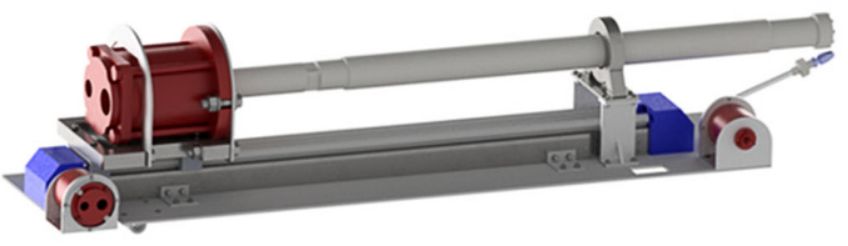

b

d
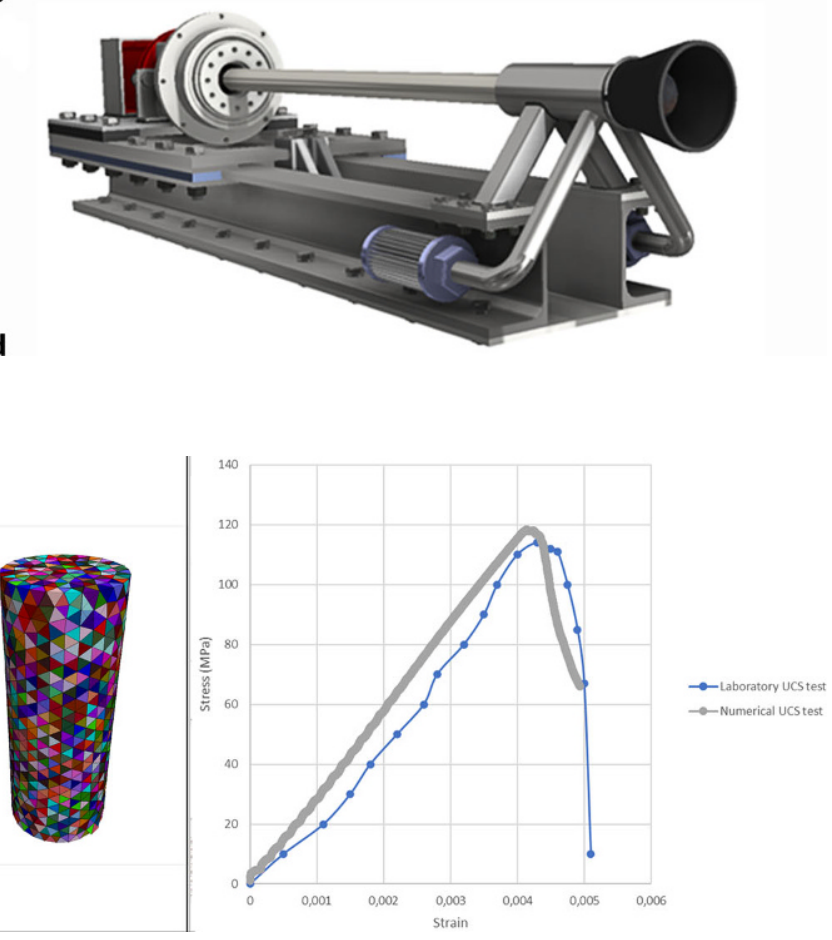

Effizienz des mechanischen Abbausystems zu verstärken $[5,6,16]$.

Die in der Praxis am häufigsten eingesetzten Abbaumethoden benötigen in mindestens einem Arbeitsschritt ein mechanisches Abbauwerkzeug. Aus diesem Grund ist es notwendig, diese Systeme genauer zu untersuchen. Die hohen Massen der mechanischen Werkzeuge im Verhältnis zur Masse der Maschine/des Roboters stellen eine zusätzliche Herausforderung dar. Daher werden, obwohl sie durch hohe spezifische Energien gekennzeichnet sind, auch alternative Abbautechnologien in Betracht gezogen, da die erforderlichen Kräfte des Abbauwerkzeugs zum Vordringen in das Gestein vergleichsweise gering sind.

In [6] wurde in Untersuchungen die Anwendbarkeit der Technologien anhand folgender Parameter bewertet:

\section{- Spezifische Energie \\ - Förderrate \\ - Vortriebskraft/Reaktionskraft}

Leistung, Masse und die Fähigkeit, die Reaktionskräfte aufzunehmen, werden als die wichtigsten Parameter ermittelt, die die Entwicklung eines kleinen Abbauwerkzeugs beeinflussen. In diesem Fall werden die Leistung und die Masse des Roboters mit $40 \mathrm{~kW}$ und $1500 \mathrm{~kg}$ angenommen. Die Be- 


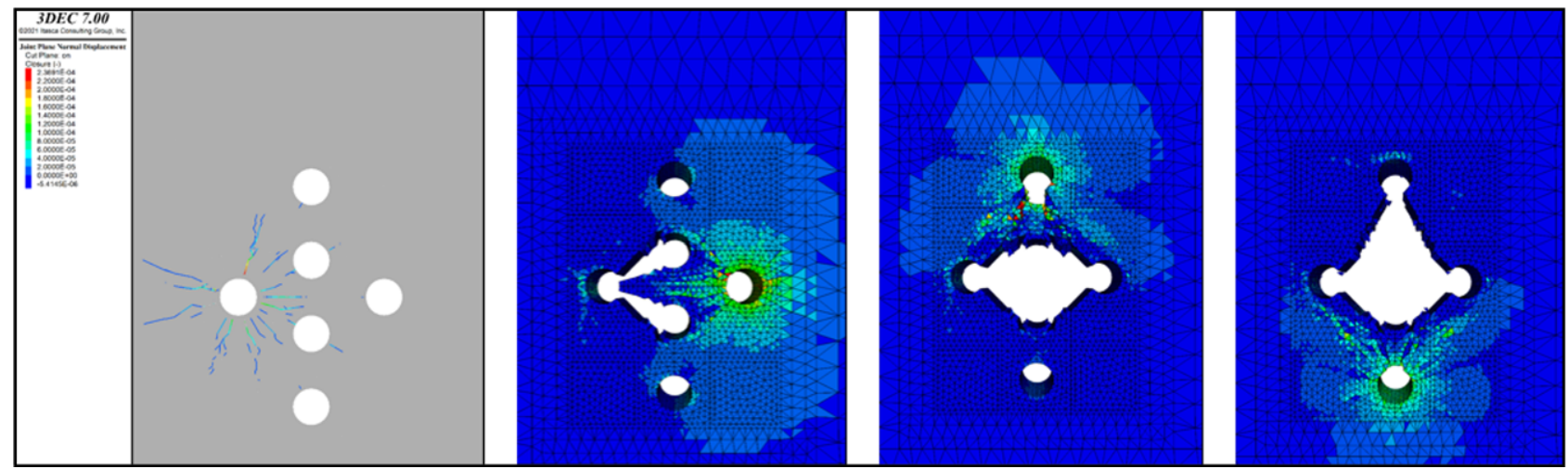

Abb. 9: Simulation eines hydrostatischen Abbauprozesses mittels DEM

schaffenheit und die Festigkeit des abzubauenden Gesteins bestimmen in hohem Maße die Effizienz des Abbauprozesses und setzen vor allem strenge Randbedingungen für jedes Abbausystem. In der Theorie wirken die erzielten Abschätzungen von spezifischen Energien und Produktionsraten vernünftig und lassen eine mögliche Machbarkeit einer bestimmten Technologie vermuten. Allerdings geben diese Ergebnisse keine Auskunft über die praktische Durchführbarkeit. In der Realität muss die Maschine/der Roboter die für das Eindringen in das Gestein erforderlichen Kräfte erzeugen - oder andersherum - mit den auf sie einwirkenden Reaktionskräften umgehen. In der Regel wird den Reaktionskräften durch die hohe Masse der Maschine und, je nach Anwendung und Konstruktion, durch zusätzliche Verankerungsmechanismen entgegengewirkt.

Wie in Abschn. 2 erörtert, übersteigen bereits die minimalen Schneidkräfte von Vollschnitttechnologien, welche bei dem Abbau von sehr weichem Gestein entstehen, die Kapazitäten des Roboters. Teilschnittmethoden können ausschließlich für weiches Gesteinsmaterial eingesetzt werden. Mit hydraulischen und hydrostatischen Abbauverfahren kann härteres Gestein bearbeitet werden, wobei alternative Methoden höhere spezifische Energien und geringere Produktionsraten aufweisen. Hochdruckwasserstrahlen senken zwar die erforderlichen Schneidkräfte der mechanischen Abbauverfahren, erfordern aber auch einen höheren Energieeinsatz. In dieser Größenordnung ist Bohren und Sprengen aufgrund der geringen Kräfte des Bohrprozesses und der hohen Effizienz des Sprengvortriebs die einzig verbleibende Option für die Gewinnung von hartem Gestein. Die technische Machbarkeit ist in diesem Schritt von untergeordneter Bedeutung und wird im Rahmen von prospektiven Aktivitäten abgedeckt.

Aufgrund der vergleichsweise geringen notwendigen Kräfte alternativer Abbaumethoden besitzen diese ein hohes Potenzial für den Einsatz in zukünftigen BergbauRobotern.

\section{Konzepte und Simulationen}

Bohren und Sprengen sowie hydraulische und hydrostatische Abbaumethoden verwenden jeweils im ersten Schritt des Abbauprozesses ein Bohrgerät zur Herstellung der
Bohrlöcher. In der Praxis werden je nach Anwendungsfall unterschiedliche Typen von Bohrgeräten verwendet. Der große Vorteil von Bohrgeräten sind die vergleichsweise geringen Vorschubkräfte, welche für den Bohrprozess benötigt werden. Hydraulische und hydrostatische Abbauverfahren sind alternative Abbaumethoden, welche als Primärwerkzeug ein Bohrgerät verwenden und als sekundäres Abbauwerkzeug den hydraulischen Druck von Fluiden verwenden.

In diesem Abschnitt werden eine Konzeptidee dieses Abbauverfahrens sowie die dazugehörige Simulationsmethodik vorgestellt. Im ersten Schritt wurden Konzepte diverser Bohrgeräte für einen Roboter $(40 \mathrm{~kW}$ Leistung und $1500 \mathrm{~kg}$ Eigengewicht) entwickelt, welche hydraulisch oder wasserhydraulisch betrieben werden. In Abb. 7 sind folgende Konzepte ersichtlich: a) hydraulischer Außenhammer mit Kettenvorschub, b) wasser-hydraulischer Imloch-Bohrhammer mit Kettenvorschub, c) wasser-hydraulischer Rotationsbohrer mit Vorschubzylinder, d) wasser-hydraulischer Rotationsbohrer mit Vorschubzylinder und Materialauffangfilter.

Im nächsten Schritt wird im Inneren der Bohrlöcher mittels eines Fluids ein Überdrück erzeugt und durch Initiierung und Fortschritt von Rissen das Gestein gelöst. Der Abbauprozess wird mithilfe der Diskrete-Elemente-Methode simuliert, um Rückschlüsse hinsichtlich der Anwendbarkeit und Effizienz der Methoden schließen zu können. Um das Gestein realitätsgetreu abzubilden, wurden im ersten Schritt die Materialparameter mittels Abgleich der Ergebnisse zweier Laborversuche kalibriert. Die Druckfestigkeit des Gesteins wird durch einen einachsigen Druckversuch (Uniaxial Compressive Strength Test - UCS Test) und die Zugfestigkeit wird durch einen indirekten Zugversuch (Brazilian Tensile Strength Test - BTS Test) ermittelt. Diese beiden Versuche wurden durch geeignete DEM Simulationen nachgebildet und die Materialparameter wurden kalibriert, um das Materialverhalten bis hin zum Versagen abzubilden. Ein Vergleich der Ergebnisse von Simulation und Prüfung der Druckfestigkeit mittels UCS Test ist in Abb. 8 ersichtlich.

Anschließend wurde eine Simulationsmethodik erstellt, womit die Auswirkungen von mit Druck beaufschlagten Bohrlöchern auf das umliegende Gestein und die Fähigkeit des Gesteinslösens untersucht wurden. Ziel dieser Simulationen ist es, Aussagen über kritische Bohrlochtiefen sowie -abstände zu treffen und Schwellwerte für hydrau- 
lische Drücke innerhalb der Bohrlöcher festzulegen. Im ersten Schritt konnten bereits Rissinitiierungs- und Rissfortschrittsphasen erfolgreich abgebildet werden. Weiters wurden mit bereits kalibrierten Materialen Simulationen mit unterschiedlichen hydraulischen Drücken durchgeführt, und es konnten Einflüsse des Drucks auf das Versagen des Gesteins entdeckt werden.

In Abb. 9 ist der Simulationsvorgang eines hydraulischen Abbauprozesses abgebildet.

Diese, zu diesem Stand noch simplifizierten, Simulationen werden in Zukunft erweitert, um auch die dynamische Charakteristik dieses Vorgangs abzubilden und in weiterer Folge die Ergebnisse für die Konzeptentwicklung zukünftiger Abbautechnologien für kleine Bergbauroboter zu verwenden.

Förderung. This project has received funding from the European Union's Horizon 2020 research and innovation programme under grant agreement No. 820971.

Funding. Open access funding provided by Montanuniversität Leoben.

Open Access Dieser Artikel wird unter der Creative Commons Namensnennung 4.0 International Lizenz veröffentlicht, welche die Nutzung, Vervielfältigung, Bearbeitung, Verbreitung und Wiedergabe in jeglichem Medium und Format erlaubt, sofern Sie den/die ursprünglichen Autor(en) und die Quelle ordnungsgemäß nennen, einen Link zur Creative Commons Lizenz beifügen und angeben, ob Änderungen vorgenommen wurden.

Die in diesem Artikel enthaltenen Bilder und sonstiges Drittmaterial unterliegen ebenfalls der genannten Creative Commons Lizenz, sofern sich aus der Abbildungslegende nichts anderes ergibt. Sofern das betreffende Material nicht unter der genannten Creative Commons Lizenz steht und die betreffende Handlung nicht nach gesetzlichen Vorschriften erlaubt ist, ist für die oben aufgeführten Weiterverwendungen des Materials die Einwilligung des jeweiligen Rechteinhabers einzuholen.

Weitere Details zur Lizenz entnehmen Sie bitte der Lizenzinformation auf http://creativecommons.org/licenses/by/4.0/deed.de.

\section{Literatur}

1. Robert, H.: Taking a step into the robotic future (2020). https://www. miningmagazine.com/innovation/news/1387411/taking-step-intothe-robotic-future, Zugegriffen: 6. Dez. 2021
2. Siciliano, B., Khatib, O. (Hrsg.): Springer Handbook of Robotics, 2. Aufl. Springer, Berlin (2016)

3. Berner, M., Sifferlinger, N.A.: H2020 - ROBOMINERS. Berg Huettenmaenn Monatsh 166(2), 59-63 (2021). https://doi.org/10.1007/ s00501-020-01074-y

4. Sifferlinger, N.A.: Roboter im Bergbau - wo liegt der Bedarf? Berg Huettenmaenn Monatsh 166(2), 53-58 (2021). https://doi.org/10. 1007/s00501-021-01079-1

5. Sifferlinger, N.A., Hartlieb, P., Moser, P.: The importance of research on alternative and hybrid rock extraction methods. Berg Huettenmaenn Monatsh 162(2), 5-8912 (2017). https://doi.org/10.1007/ s00501-017-0574-y

6. Berner, M., Sifferlinger, N.A.: Analysis of excavation methods for a small-scale mining robot. In: Osumi, H. (Hrsg.) Proceedings of the 37th International Symposium on Automation and Robotics in Construction (ISARC). International Association for Automation and Robotics in Construction (IAARC), (2020)

7. Evans, I.: A theory of the cutting force for point-attack picks. Int. J. Min. Eng. 2(1), 63-71 (1984). https://doi.org/10.1007/BF00880858

8. Göktan, R.M.: Effect of cutter pick rake angle on the failure pattern of high-strength rocks. Min. Sci. Technol. 11(3), 281-285 (1990). https:// doi.org/10.1016/0167-9031(90)90981-W

9. Goktan, R.M., Gunes, N.: A semi-empirical approach to cutting force prediction for point-attack picks. J. S. Afr. Inst. Min. Metall. 105(4), 257-263 (2005)

10. Roxborough, F.F., Liu, Z.C.: Theoretical considerations on pick shape in rock and coal cutting. In: Proceedings of the Sixth Underground Operator's Conference, S. 189-193. (1995)

11. Rostami, J., Ozdemir, L.: A New Model for Performance Prediction of Hard Rock Tbms (1997)

12. Kunze, G., Göhring, H., Jacob, K.: Baumaschinen. Erdbau- und Tagebaumaschinen; mit 650 Abbildungen und 147 Tabellen, 2. Aufl. Maschinenelemente und Konstruktion. Vieweg + Teubner, Wiesbaden (2012)

13. WPFX: Track vs wheeled equipment: which type of machine should I rent? (2017). https://www.macallisterrentals.com/track-vs-wheeledequipment-type-machine-rent/, Zugegriffen: 15. Dez. 2021

14. Cole, B.N.: Inquiry into amphibious screw traction. Proc. Inst. Mech. Eng. 175(1), 919-940 (1961). https://doi.org/10.1243/PIME_PROC_ 1961_175_060_02

15. Vogt, D.: A review of rock cutting for underground mining: past, present, and future. J. S. Afr. Inst. Min. Metall. 116(11), 1011-1026 (2016). https://doi.org/10.17159/2411-9717/2016/v116n11a3

16. Bilgin, N.: Mechanical Excavation in Mining and Civil Industries. Taylor \& Francis, Baton Rouge (2014)

Hinweis des Verlags. Der Verlag bleibt in Hinblick auf geografische Zuordnungen und Gebietsbezeichnungen in veröffentlichten Karten und Institutsadressen neutral. 